Журнал обчислювальної та

прикладної математики
Journal of Numerical

\& Applied Mathematics

УДК 519.711.3

MSC 90C31, 93C55, 93C95, 03C45, 03H10

\title{
METHODS OF OPTIMIZATION OF PARAMETRIC SYSTEMS
}

\author{
V.T. Matvienko, V.V. Pichkur, D.I. Cherniy
}

Faculty of Computer Science and Cybernetics, Taras Shevchenko National University of Kyiv, Kyiv, Ukraine, E-mail: vpichkur@gmail.com,d_cherniy@gmail.com

\section{МЕТОДИ ОПТИМІЗАЦІї ПАРАМЕТРИЧНИХ СИСТЕМ}

\author{
В.Т. МАтвієнко, В.В. ПгчкУР, Д.І. ЧЕРній
}

Факультет комп'ютерних наук та кібернетики, Київський національний університет імені Тараса Шевченка, Київ, Україна, E-mail: vpichkur@gmail.com, d_cherniy@gmail.com

ABstract. The paper considers methods of parametric optimization of a dynamical system, which is described by a parametric system of differential equations. The gradient of the functional in the form of Boltz is found, which is the basis of methods such as gradient descent. Another method is based on the application of the sensitivity function.

KEYWORDS: parametric optimization, sensitivity matrix, optimality conditions.

АнотАція. В роботі розглядаються методи параметричної оптимізації динамічної системи, яка описується параметричною системою диференціальних рівнянь. Знайдено градієнт функціоналу у формі Больца, який є основою методів типу градієнтного спуску. Розгляд метод заснований на застосуванні функції чутливості.

КлЮчовІ СловА: параметрична оптимізація, матриця чутливості, умови оптимальності.

\section{ВСТУП}

Аналіз проблем математичного моделювання, а також прикладних задач, пов'язаних із конструюванням складних технічних систем, показує, що побудова математичної моделі здійснюється в параметрично заданому класі $[1,2]$. Вибір параметрів математичної моделі відображає такі її характеристики як адекватність і оптимальність. Математично це означає, що виникає задача знаходження оптимальних допустимих параметрів системи у заданому класі, які мінімізують деякий критерій якості, що описує відповідну якість функціонування системи. У такий спосіб виникає проблема параметричної оптимізації системи [1-4]. Зокрема, аналогічна проблематика виникає в задачах, пов'язаних з оптимізацією структури систем прискорення і фокусування $[1,2]$. 
В статті ми пропонуємо один з підходів до розв'язування задач параметричної оптимізації систем, який базується на застосуванні варіаційного методу. Варіаційний метод дозволяє побудувати градієнт за параметрами від критерія якості на розв'язках параметричної системи і конструювати ітераційні процедури типу градієнтного спуску за обраними параметрами [1]. Разом з тим, для збільшення ефективності обчислень виникає необхідність у побудові методів, які близькі до методів другого порядку. Один з таких методів, який описується в роботі, базується на властивостях функції чутливості і використовує підходи, характерні для методу Ньютона [5].

\section{1. ПАРАМЕТРИЧНА ОПТИМІЗАЦІЯ ДИНАМІЧНИХ СИСТЕМ. НЕЛІНІЙНИЙ ВИПАДОК}

Розглянемо задачу параметричної оптимізації динамічної системи

$$
\mathscr{I}(p)=\int_{t_{0}}^{T} f_{0}(x(t), p, t) d t+\Phi(x(T)) \rightarrow \inf
$$

за умов, що

$$
\frac{d x(t)}{d t}=f(x(t), p, t), x\left(t_{0}\right)=x_{0}, t \in\left[t_{0}, T\right] .
$$

Тут $x=\left(x_{1}, x_{2}, \ldots, x_{n}\right)$ - вектор стану, $p=\left(p_{1}, p_{2}, \ldots, p_{m}\right)$ - параметр, за яким здійснюється оптимізація, $f_{0}(x, p, t)$ - неперервно диференційована функція за $x$ і $p, f(x, p, t)=\left(f_{1}(x, p, t), \ldots, f_{n}(x, p, t)\right)^{*}$ - неперервно диференційована функція за $x$ та $p$. Припустимо, що параметр $p_{*} \in$ оптимальним, тобто, він доставляє мінімум функціоналу (1).

Нехай $h \in \mathbb{R}^{m}$ - напрямок, за яким ми будемо здійснювати варіацію функціоналу (1). Позначимо

$$
\varphi(\alpha)=\mathscr{I}\left(p_{*}+\alpha h\right)
$$

і запишемо необхідну умову екстремуму через першу варіацію функціоналу

$$
\varphi^{\prime}(0)=\delta \mathscr{I}\left(p_{*}, h\right)=0 .
$$

Параметру $p=p_{*}+\alpha h$ відповідає траєкторія $x(t)=x(t, \alpha)$ системи $(2)$. Тоді

$$
\begin{gathered}
\varphi^{\prime}(0)=\int_{t_{0}}^{T}\left\{\left\langle\frac{\partial f_{0}(x(t), p, t)}{\partial x}, \frac{\partial x(t)}{\partial \alpha}\right\rangle+\right. \\
\left.+\left\langle\frac{\partial f_{0}(x(t), p, t)}{\partial p}, h\right\rangle\right\} d t+\left\langle\frac{\partial \Phi(x(T))}{\partial x}, \frac{\partial x(T)}{\partial \alpha}\right\rangle .
\end{gathered}
$$

Тут похідна $\frac{\partial x(t)}{\partial \alpha}$ береться в точці $\alpha=0$. Позначимо $\frac{\partial x(t)}{\partial \alpha}=z(t)$. Маємо рівняння в варіаціях

$$
\frac{d z(t)}{d t}=\frac{\partial f\left(x(t), p_{*}, t\right)}{\partial x} z(t)+\frac{\partial f\left(x(t), p_{*}, t\right)}{\partial p} h, z\left(t_{0}\right)=0 .
$$


XX Міжнародний симпозіум «Методи дискретних особливостей в задачах математичної фізики/Discrete Singularities Methods in Mathematical Physics»,

Тоді

$$
\begin{aligned}
& \varphi^{\prime}(0)=\int_{t_{0}}^{T}\left\{\left\langle\frac{\partial f_{0}\left(x(t), p_{*}, t\right)}{\partial x}, z(t)\right\rangle+\right. \\
& \left.+\left\langle\frac{\partial f_{0}\left(x(t), p_{*}, t\right)}{\partial p}, h\right\rangle\right\} d t+\left\langle\frac{\partial \Phi(x(T))}{\partial x}, z(T)\right\rangle .
\end{aligned}
$$

Введемо спряжені змінні $\psi=\left(\psi_{1}, \psi_{2}, \ldots, \psi_{n}\right)^{*}$. Нехай

$$
\psi(T)=-\frac{\partial \Phi(x(T))}{\partial x} .
$$

Враховуючи рівність (3), одержуємо

$$
\begin{gathered}
\left\langle\frac{\partial \Phi(x(T))}{\partial x}, z(T)\right\rangle=-\langle\psi(T), z(T)\rangle=-\left(\langle\psi(T), z(T)\rangle-\left\langle\psi\left(t_{0}\right), z\left(t_{0}\right)\right\rangle\right)= \\
-\int_{t_{0}}^{T} d\langle z(t), \psi(t)\rangle==-\int_{t_{0}}^{T}\left\{\left\langle\frac{d z(t)}{d t}, \psi(t)\right\rangle+\left\langle\frac{d \psi(t)}{d t}, z(t)\right\rangle\right\} d t .
\end{gathered}
$$

Підставляємо останній вираз в (4). Маємо

$$
\begin{gathered}
\varphi^{\prime}(0)=\int_{t_{0}}^{T}\left\{\left\langle\frac{\partial f_{0}\left(x(t), p_{*}, t\right)}{\partial x}-\frac{d \psi(t)}{d t}, z(t)\right\rangle-\right. \\
\left.-\left\langle\frac{d z(t)}{d t}, \psi(t)\right\rangle+\left\langle\frac{\partial f_{0}\left(x(t), p_{*}, t\right)}{\partial p}, h\right\rangle\right\} d t .
\end{gathered}
$$

Враховуємо рівняння (3). Отримуємо таке співвідношення

$$
\begin{aligned}
\varphi^{\prime}(0)=\int_{t_{0}}^{T} & \left\{\left\langle\frac{\partial f_{0}\left(x(t), p_{*}, t\right)}{\partial x}-\frac{d \psi(t)}{d t}, z(t)\right\rangle-\left\langle\frac{\partial f\left(x(t), p_{*}, t\right)}{\partial x} z(t), \psi(t)\right\rangle-\right. \\
- & \left.\left\langle\frac{\partial f\left(x(t), p_{*}, t\right)}{\partial p} h, \psi(t)\right\rangle+\left\langle\frac{\partial f_{0}\left(x(t), p_{*}, t\right)}{\partial p}, h\right\rangle\right\} d t= \\
=\int_{t_{0}}^{T} & \left\{\left\langle\frac{\partial f_{0}\left(x(t), p_{*}, t\right)}{\partial x}-\frac{\partial f^{*}\left(x(t), p_{*}, t\right)}{\partial x} \psi(t)-\frac{d \psi(t)}{d t}, z(t)\right\rangle+\right. \\
& \left.+\left\langle-\frac{\partial f^{*}\left(x(t), p_{*}, t\right)}{\partial p} \psi(t)+\frac{\partial f_{0}\left(x(t), p_{*}, t\right)}{\partial p}, h\right\rangle\right\} d t .
\end{aligned}
$$

Тоді накладаємо на функцію $\psi(t)$ умову

$$
\frac{\partial f_{0}\left(x(t), p_{*}, t\right)}{\partial x}-\frac{\partial f^{*}\left(x(t), p_{*}, t\right)}{\partial x} \psi(t)-\frac{d \psi(t)}{d t}=0 .
$$

У такий спосіб

$$
\begin{aligned}
& \varphi^{\prime}(0)=-\int_{t_{0}}^{T}\left\{\left\langle\frac{\partial f^{*}\left(x(t), p_{*}, t\right)}{\partial p} \psi(t)-\frac{\partial f_{0}\left(x(t), p_{*}, t\right)}{\partial p}, h\right\rangle\right\} d t= \\
& =-\left\langle\int_{t_{0}}^{T}\left\{\frac{\partial f^{*}\left(x(t), p_{*}, t\right)}{\partial p} \psi(t)-\frac{\partial f_{0}\left(x(t), p_{*}, t\right)}{\partial p}\right\} d t, h\right\rangle .
\end{aligned}
$$

Введемо функцію Гамільтона-Понтрягіна

$$
\mathscr{H}(x, p, \psi, t)=-f_{0}(x, p, t)+\langle\psi, f(x, p, t)\rangle .
$$


Тоді з умови (5) випливає система для знаходження спряженої функції

$$
\frac{d \psi(t)}{d t}=-\frac{\partial \mathscr{H}\left(x(t), p_{*}, \psi(t), t\right)}{\partial x}, \psi(T)=-\frac{\partial \Phi(x(T))}{\partial x} .
$$

3 (6) знаходимо варіацію функціоналу

$$
\varphi^{\prime}(0)=-\left\langle\int_{t_{0}}^{T} \frac{\partial \mathscr{H}\left(x(t), p_{*}, \psi(t), t\right)}{\partial p} d t, h\right\rangle .
$$

Отже,

$$
\mathscr{I}^{\prime}\left(p_{*}\right)=-\int_{t_{0}}^{T} \frac{\partial \mathscr{H}\left(x(t), p_{*}, \psi(t), t\right)}{\partial p} d t .
$$

Алгоритм параметричної оптимізації може бути реалізований на основі методу градієнтного спуску [6]

$$
p(k+1)=p(k)-\rho_{k} \mathscr{I}^{\prime}(p(k)), k=0,1, \ldots
$$

Крок $\rho>0$ доцільно вибирати, згідно методу найшвидшого спуску, як роз'язок задачі

$$
\min _{\rho \in(0, R]} \mathscr{I}\left(p(k)-\rho \mathscr{I}^{\prime}(p(k))\right),
$$

де $R>0$ - параметр методу [6].

Якщо на керування задано обмеження у вигляді $p \in \mathscr{P}$, де $\mathscr{P} \in \operatorname{conv}\left(\mathbb{R}^{m}\right)$, тоді одержуємо варіаційну нерівність

$$
\left\langle\int_{t_{0}}^{T} \frac{\partial \mathscr{H}\left(x(t), p_{*}, \psi(t), t\right)}{\partial p} d t, p-p_{*}\right\rangle \leq 0 \forall p \in \mathscr{P} .
$$

Розглянемо приклад. Розв'яжемо задачу мінімізації функціоналу

$$
\mathscr{I}(p)=\frac{1}{2} \int_{t_{0}}^{T}\{\langle N(t) x(t), x(t)\rangle+\langle M(t) p, p\rangle\} d t+\frac{1}{2}\left\langle P_{0} x(T), x(T)\right\rangle
$$

за умови, що

$$
\frac{d x(t)}{d t}=A(t) x(t)+C(t) p, x\left(t_{0}\right)=x_{0} .
$$

Тут $N(t), P_{0}$ - невід'ємновизначені симетричні матриці розмірності $n \times n$, $M(t)$ - додатновизначена симетрична матриця розмірності $m \times m, A(t)$ - $n \times n$-матриця 3 неперервними компонентами, $C(t)-n \times m$-матриця 3 неперервними компонентами, $t \in\left[t_{0}, T\right]$.

Припустимо, що параметр $p_{*}$ доставляє мінімум квадратичному функціоналу. Введемо функцію Гамільтона-Понтрягіна

$$
\mathscr{H}(x, p, \psi, t)=-\frac{1}{2}\{\langle N(t) x(t), x(t)\rangle+\langle M(t) p, p\rangle\}+\langle\psi, A(t) x+C(t) p\rangle .
$$

Спряжена система у цьому випадку має вигляд

$$
\frac{d \psi(t)}{d t}=-A^{*}(t) \psi(t)+N(t) x(t), \psi(T)=-P_{0} x(T) .
$$

Необхідна умова екстремуму

$$
\mathscr{I}^{\prime}(p)=-\int_{t_{0}}^{T} \frac{\partial \mathscr{H}(x(t), p, \psi(t), t)}{\partial p} d t=0 .
$$


XX Міжнародний симпозіум «Методи дискретних особливостей в задачах математичної фізики/Discrete Singularities Methods in Mathematical Physics»,

У такий спосіб отримуємо рівність

$$
\int_{t_{0}}^{T}\left\{M(t) p-C^{*}(t) \psi(t)\right\} d t=0 .
$$

Звідси маємо

$$
\int_{t_{0}}^{T} M(t) d t p=\int_{t_{0}}^{T} C^{*}(t) \psi(t) d t .
$$

Отже, розв'язком задачі є точка

$$
p_{*}=\left(\int_{t_{0}}^{T} M(t) d t\right)^{-1} \int_{t_{0}}^{T} C^{*}(t) \psi(t) d t .
$$

\section{2. МЕТОД ПАРАМЕТРИЧНОЇ ОПТИМІЗАЦІЇ НА ОСНОВІ МАТРИЦІ чУтливості}

Розглянемо динамічну систему (2). Матрицею чутливості системи (2) в точці $p=\hat{p}$ називається функція, яка задається співвідношенням $U(t)=$ $\frac{\partial x(t, \hat{p})}{\partial p}$. Тут $x(t)=x(t, \hat{p})$ є розв'язком системи (2) при $p=\hat{p}$ [5]. $3(2)$ отримуємо еквівалентне інтегральне рівняння

$$
x(t, p)=x_{0}+\int_{t_{0}}^{t} f(x(s), p, s) d s .
$$

Диференціюємо останню рівність за змінною $p$ і підставляємо $p=\hat{p}$. Отримуємо інтегральне співвідношення для знаходження матриці чутливості

$$
U(t)=\int_{t_{0}}^{t}\left(\frac{\partial f(x(s), \hat{p}, s)}{\partial x} U(s)+\frac{\partial f(x(s), \hat{p}, s)}{\partial p}\right) d s .
$$

Продиференціюємо останню рівність. Отже, матриця чутливості задовольняє матричне диференціальне рівняння

$$
\frac{d U(t)}{d t}=\frac{\partial f(x(t), \hat{p}, t)}{\partial x} U(t)+\frac{\partial f(x(s), \hat{p}, t)}{\partial p}, U\left(t_{0}\right)=0 .
$$

Отримуємо $x(t, p)=x(t, \hat{p})+U(t) h+v(t, h), p=\hat{p}+h$. Тут $\lim _{h \rightarrow 0} \frac{v(t, h)}{\|h\|}=0$.

Розглянемо критерій якості (7) на розв'язках динамічної системи (2). Одержимо $\mathscr{I}(p)=\mathscr{I}(\hat{p}+h)$. Задача полягає у виборі $h$, що мінімізує $\mathscr{I}(\hat{p}+h)$. Підставляємо $p=\hat{p}+h$ і $x(t, p)=x(t, \hat{p})+U(t) h+v(t, h)$ в (7). Одержуємо

$$
\begin{gathered}
\mathscr{I}(\hat{p}+h)= \\
=\frac{1}{2} \int_{t_{0}}^{T}\{\langle N(t)(x(t, \hat{p})+U(t) h), x(t, \hat{p})+U(t) h\rangle+\langle M(t)(\hat{p}+h), \hat{p}+h\rangle\} d t+ \\
\quad+\frac{1}{2}\left\langle P_{0}(x(T, \hat{p})+U(T) h), x(T, \hat{p})+U(T) h\right\rangle=\mathscr{Q}(h)+w(h) .
\end{gathered}
$$


Тут

$$
\begin{gathered}
\mathscr{Q}(h)=\frac{1}{2}\langle R h, h\rangle+\langle g, h\rangle+r \\
R=\int_{t_{0}}^{T}\left(U^{*}(t) N(t) U(t)+M(t)\right) d t+U^{*}(T) P_{0} U(T), \\
g=\int_{t_{0}}^{T}\left(U^{*}(t) N(t) x(t, \hat{p})+M(t) \hat{p}\right) d t+U^{*}(T) P_{0} x(T, \hat{p}), \\
r=\frac{1}{2} \int_{t_{0}}^{T}(\langle N(t) x(t, \hat{p}), x(t, \hat{p})\rangle+\langle M(t) \hat{p}, \hat{p}\rangle) d t+\frac{1}{2}\left\langle P_{0} x(T, \hat{p}), x(T, \hat{p})\right\rangle,
\end{gathered}
$$

$\lim _{h \rightarrow 0} \frac{w(h)}{\|h\|}=0$. Оптимальне значення $h$ шукаємо з умови екстремуму квадратичної форми $\mathscr{Q}(h)=\frac{1}{2}\langle R h, h\rangle+\langle g, h\rangle+r$. 3 умови $\frac{d \mathscr{Q}(h)}{d h}=0$ випливає $R h+g=0$. Звідси $h_{*}=-R^{+} g$, де $R^{+}$- псевдообернена матриця до матриці $R$. У такий спосіб приходимо до такого алгоритму.

Задаємо початкові данні: матриці $N(t), M(t), P_{0}$, праву частину системи $(2)$, точку $x_{0} \in \mathbb{R}^{n}$, систему для матриці чутливості (8), часовий інтервал $t \in\left[t_{0}, T\right]$ і початкове наближення параметра $p=p^{(0)}$.

Крок 1. Знаходимо роз'язок $x\left(t, p^{(0)}\right)$ системи $(2)$ і підставляємо його і $p=p^{(0)}$ в систему (8). Знаходимо матрицю чутливості $U_{0}(t)$.

Крок 2. Обчислюємо

$$
\begin{gathered}
R_{0}=\int_{t_{0}}^{T}\left(U_{0}^{*}(t) N(t) U_{0}(t)+M(t)\right) d t+U_{0}^{*}(T) P_{0} U_{0}(T), \\
g_{0}=\int_{t_{0}}^{T}\left(U_{0}^{*}(t) N(t) x\left(t, p^{(0)}\right)+M(t) p^{(0)}\right) d t+U_{0}^{*}(T) P_{0} x\left(T, p^{(0)}\right) .
\end{gathered}
$$

Далі визначаємо $h_{0}=-R_{0}^{+} g_{0}$ і наступне наближення $p^{(1)}=p^{(0)}+h_{0}$.

Крож 3. Нехай знайшли $p=p^{(k)}$. Тоді обчислюємо роз'язок $x\left(t, p^{(k)}\right)$ системи $(2),(? ?)$ і підставляємо його $x\left(t, p^{(k)}\right), p=p^{(k)}$ в систему (8). Знаходимо матрицю чутливості $U_{k}(t)$.

Крок 3. Обчислюемо

$$
\begin{gathered}
R_{k}=\int_{t_{0}}^{T}\left(U_{k}^{*}(t) N(t) U_{k}(t)+M(t)\right) d t+U_{k}^{*}(T) P_{0} U_{k}(T), \\
g_{k}=\int_{t_{0}}^{T}\left(U_{k}^{*}(t) N(t) x\left(t, p^{(0)}\right)+M(t) p^{(k)}\right) d t+U_{k}^{*}(T) P_{0} x\left(T, p^{(k)}\right) .
\end{gathered}
$$

Далі визначаємо $h_{k}=-R_{k}^{+} g_{k}$ і наступне наближення

$$
p^{(k+1)}=p^{(k)}+h_{k} .
$$

Крок 4. Одна з можливих умов зупинки алгоритму така:

$$
\left|\mathscr{I}\left(p^{(k+1)}\right)-\mathscr{I}\left(p^{(k)}\right)\right|<\epsilon,
$$

156 де $\epsilon>0$ - наперед задане число. Кінець опису алгоритма.

Алгоритм допускає різні модифікації, характерні для метода Ньютона. 
XX Міжнародний симпозіум «Методи дискретних особливостей в задачах математичної фізики/Discrete Singularities Methods in Mathematical Physics», МДОЗМФ/DSMMPh-2021

\begin{abstract}
Висновок
Розглянуто методи параметричної оптимізації динамічної системи, яка описується параметричною системою диференціальних рівнянь. Знайдено градієнт функціоналу у формі Больца, який є основою методів типу градієнтного спуску. Для знаходження градієнту необхідно послідовно знаходити розв'язки системи і спряженої системи, які відповідають поточному наближенню параметра, а також розв'язувати крайову задачу. Це дозволяє розвивати для таких задач ітераційні методи типу метода градієнтного спуска. Ще один метод базується на властивостях матриці чутливості. При цьому значення критерія якості авроксимується квадратичною формою, що дозволяє одержати наступне наближення мараметра аналітично.
\end{abstract}

\title{
ЛIтерАТУРА
}

1. Бублик Б.Н., Гаращенко Ф.Г., Кириченко Н.Ф. Структурно-параметрическая оптимизация и устойчивость динамики пучков. К.: Наукова думка, 1985.

2. Гаращенко Ф.Г., Панталієнко Л.А. Аналіз та оцінка параметричних систем. К.: ІСДО, 1995.

3. Башняков О.М., Гаращенко Ф.Г., Пічкур В.В. Практична стійкість та структурна оптимізація динамічних систем. К.: ВПЦ “Київський університет”. 2000.

4. Башняков О.М., Гаращенко Ф.Г., Пічкур В.В. Практична стійкість, оцінки та оптимізація. К.: Київський університет. 2008.

5. Розенвассер Е.Н., Юсупов Р.М. Чувствительность систем управления. М.: Наука, 1981.

6. Бейко И.В., Бублик Б.Н., Зинько П.Н. Методы и алгоритмы решения задач оптимизации. К.: Вища школа, 1983.

Надійшла: 30.05.2021.

\section{МЕТОДЫ ОПТИМИЗАЦИИ ПАРАМЕТРИЧЕСКИХ СИСТЕМ}

\author{
В.Т. МАтвиЕнко, В.В. ПичКУР, Д.И. ЧЕРНИй
}

Факультет компьютерных наук и кибернетики, Киевский национальный университет имени Тараса Шевченко, E-mail: vpichkur@gmail.com,d_cherniy@gmail.com

АннотАция. В работе рассматриваются методы параметрической оптимизации динамической системы, которая описывается параметрической системой дифференциальных уравнений. Найден градиент функционала в форме Больца, который является основой методов типа градиентного спуска. Другой метод основан на применении функции чувствительности.

КЛЮчЕВЫЕ СЛовА: параметрическая оптимизация, матрица чувствительности, условия оптимальности. 